\title{
Demographic factors in the Chinese health-care market
}

\section{Susan Ward}

China was the fastest-growing pharmaceutical market in 2004, with growth rates of $28 \%$, and is forecasted to be the world's largest by 2050 (REF. 1). Multinational pharmaceutical companies manufactured 40 of the top 50 best-selling drugs ${ }^{2}$. In 2006, the market is estimated to be US\$13 billion, and is forecasted to grow to between $\$ 22-25$ billion by 2010 (REF. 3).

The growth of the Chinese health-care market is largely being driven by the changing age profile of the population, its rapid economic development, and urbanization. In particular, therapeutic areas such as type 2 diabetes have grown considerably in importance. Type 2 diabetes was a rare disease in China 20 years ago, but its prevalence has doubled in the past decade, with $~ 56.7$ million people affected today - a number that is almost equivalent to the total UK population and double the estimated burden in the US of 20.8 million $^{4}$. Understanding population factors such as the age profile, lifestyle changes associated with modernization, and growing treatment affordability is important in predicting how the Chinese health-care market will evolve. In this article, these issues are discussed on the basis of population data and forecasts from Global Demographics Healthcare (see BOX 1 for details).

\section{Age profile}

China's 'one child' policy (introduced in 1979), better education and increasing affluence combine to significantly affect the country's birth rate. The number of births is expected to decrease by almost one third from 12.7 million in 2007 to 8.9 million in 2026. The reduced birth rate combined with increasing life expectancy due to improved nutrition and access to health care has a dramatic effect on the population age profile of China (FIG. 1).

The number of people under 25 years of age is predicted to decline $47 \%$, from 437 million in 2007 to 297 million in 2026. At the same time, the number of people over 40 years of age is predicted to increase from 566 million people to 740 million people. Within the next decade, $50 \%$ of the population will be over 40 years of age and $25 \%$ will be over 60 years of age.
This age profile trend will significantly affect the future health profile: as the population ages, there will be a greater proportion of degenerative diseases, such as cancer, heart disease and diabetes (FIG. 2a). For example, urban adults aged $40-49$ years in China are twice as likely to have diabetes as those aged 30-39 years. And adults over the age of 60 are four-times as likely to have diabetes than those aged 30-39 years. Nearly $60 \%$ of all urban adults aged 65 year olds have hypertension, which is double the rate of a person aged 30 years, and urban females aged 55-59 years are twice as likely to develop breast cancer than females 20 years younger.

\section{Modernization}

Owing to the one-child policy, the education level has increased dramatically, as China has spent the same resources on fewer children, resulting in literacy rates of $98 \%$. Those better educated tend to seek higher-paid work in urban areas, driving urbanization and in turn workforce capability and affluence. Urbanization has increased from 32\% in 1997 to $43 \%$ today and is predicted to reach $52 \%$ in 2017. An 'affluence wave' is also occurring: the average urban household income is predicted to increase from 34,973 rmb $(\$ 4,032)$ in 2007 to $41,586 \mathrm{rmb}(\$ 5,115)$ in 2017 , and the GDP is predicted to increase from 18 trillion $\mathrm{rmb}$ ( $\$ 2.2$ trillion) to 26 trillion $\mathrm{rmb}$ ( $\$ 3.2$ trillion).

These developments have led to important lifestyle changes, such as decreased physical activity, a change in diet and increased exposure to toxins, such as tobacco and alcohol. Indeed, a threefold difference in levels of physical activity between urban and rural areas has been observed ${ }^{5}$, as well as a move to a new diet that is rich in refined carbohydrates, fats and meat, leading to increased obesity levels. Moreover, in 2002 the prevalence of overweight adults was $22.8 \%$, and $7.1 \%$ were classified as obese, compared with $6 \%$ and 0.6\%, respectively, in 1982 (REF. 6).

As physical inactivity and obesity are the two key risk factors for type 2 diabetes, the urban diabetes prevalence rates are forecasted to increase - for example, by $40 \%$ over the next decade for males between 20 and 50 years of age. So, with the rapid aging

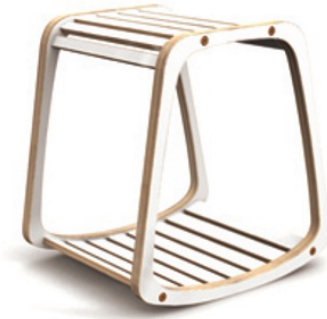

'Wednesday Evening' designed by Ryan Frank www.ryanfrank.net

and modernization, the urban prevalence of diabetes is predicted to increase from 32.7 million in 2007 to 56.3 million in 2017. This absolute increase of $76 \%$ results in the addition of 2.4 million diabetics per year. However, the low diagnosis rate is a major concern. In 2007, only 10 million or $32 \%$ of urban diabetics were diagnosed. This is in contrast to $50 \%$ in neighbouring Hong $\mathrm{Kong}^{7}$, and $70 \%$ in the US ${ }^{4}$. Furthermore, in populations where diagnosis rate is low, it is feared that the prevalence of having a complication of diabetes, such as kidney failure or heart disease at diagnosis is higher, hence the need for both earlier diagnosis and treatment of the diabetic population.

Similar increases in prevalence are anticipated for other degenerative diseases. For example, the urban prevalence of hypertension is predicted to grow from 65 million in 2005, to 100 million in 2011, and urban breast cancer incidence is predicted to increase $62 \%$ from 112,113 to 181,190 women per year.

\section{Affordability}

One key issue in China, which is typical of emerging markets, is the fact that the patient is the major payer for health care, and patient affordability is therefore a key determinant of patient access. Using diabetes as an example, FIG. 2b illustrates how this affects market value.

The expansion of the market economy in China over the past two decades has resulted in a dramatic shift away from government-funded health care to a situation in which the self-pay sector (consumer expenditure on health care) predominates, and over $50 \%$ of health-care bills are paid for out of pocket. In 1985, just less than $40 \%$ of bills were paid by the government and one-third by social health insurance. By 2005 , almost $56 \%$ of all health-care bills were paid for out of pocket, whereas the government's role had been reduced to $17 \%$ (REF. 8). The self-pay healthcare expenditure is estimated to increase from $2 \%$ to $3 \%$ of GDP in the next 10 years.

Even though there is a move to increase social health-insurance contributions, this tends to be in the format of a proportion reimbursed, and so patient affordability will 


\section{CHINESE HEALTH-CARE | MARKET INDICATORS}

- remain a key part of patient access. This is a different payer environment to the largest health-care markets - the US, Europe and Japan - where the major payer is either the government (for example, the National Health Service in the UK) or a medical insurance company in the US and Japan.

Patients that can afford treatment will be the target clients for multinational pharmaceutical companies. Patients that cannot afford treatment tend to be the elderly (who missed out on the education process owing to the Cultural Revolution and hence better paid jobs) and the rural; the opportunity for pharmaceutical companies here is with government and typically at lower treatment prices.

Susan Ward is at Global Demographics Healthcare Ltd, 25F Bank of China Tower, 1 Garden Road, Central, Hong Kong. e-mail:sward@global-dem.com doi: $10.1038 /$ nrd2527 Published online 11 April 2008

1. Ernst \& Young. Unmasking China's Pharmaceutical Future (2005).

2. China Economic Information Network [online] $<$ http://www1.cei.gov.cn/ce/doc/ceng/200403171474. htm > (2004)

3. Hill, R. China pharma dynamics. Pursuing growth in a challenging environment. The Pharmaceutical Business Research Intelligence and Research Group web site [online], < http://www.pbirg.com/new/docs/ 2007workshop/2007FallEducationWorkshop3FINALAbriged.ppt\#517,1,China\%20pharma\%20dynamics > (2007).

4. National Institute of Diabetes, Digestive and Kidney Disease (NIDDK). National Diabetes Statistics. NIDDK web site [online], < http://diabetes.niddk.nih.gov/dm/ pubs/statistics/\#7 > (2005)

5. Muntner, P. et al. Prevalence of physical activity among Chinese adults: results from the International Collaborative Study of Cardiovascular Disease in Asia. Am. J. Pub. Health 95, 1631-1636 (2005).

6. Carolina Population Center (CPC). China Health and Nutrition Survey. CPC web site [online], $<$ http://www.cpc.unc.edu/china > (2008).

7. Wong, K. C. \& Wang, Z. Prevalence of type 2 diabetes mellitus of Chinese populations in Mainland China, Hong Kong and Taiwan. Diabetes Res. Clin. Prac. 73 126-134 (2006).

8. National Bureau of Statistics of China (NBSC). China Health Yearbook 2006. NBSC web site [online], $<$ http://www.stats.gov.cn/ > (2006).

\section{Box 1 | Forecast methodology}

Forecasts used in this article are drawn from the Global Demographics Healthcare database and models, which in turn is driven by actual data published from the State Statistical Bureau, including census data for 1990 and 2000 and inter-year sample data, actual incomes and expenditure data from the Household Income and Expenditure Survey taken annually and actual epidemiology from cancer registries and published medical research from 1984 onwards. More details can be found at http://www.global-dem.com.

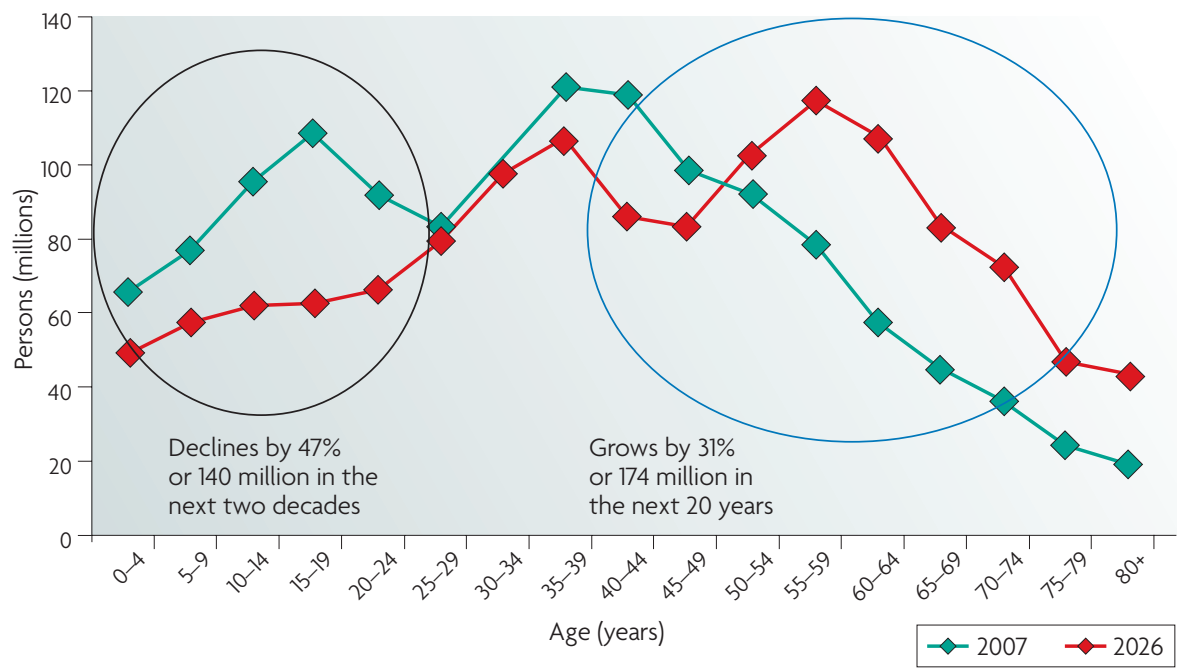

Figure 1 | Age profile trend of the population in China. In the next two decades, the proportion of people under the age of 25 will decrease, whereas the proportion over the age of 40 will increase. Source: Global Demographics Ltd.
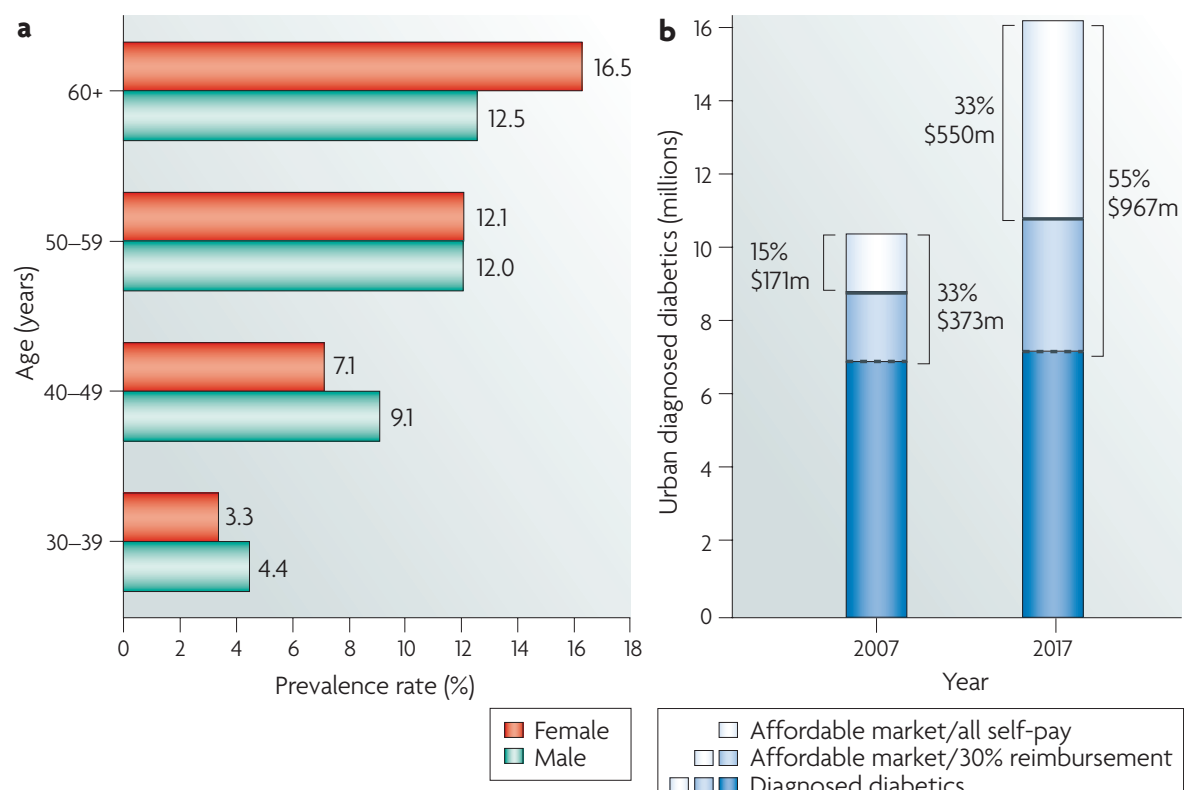

$\square$ Affordable market/all self-pay
$\square \square$ Affordable market/30\% reimbursement
$\square \square \square$ Diagnosed diabetics

Figure 2 | Diabetes in China: prevalence, market value and affordability of treatment.

a | Urban diabetic prevalence rates in 2007. b | If all patients diagnosed with diabetes had access to a treatment costing 50 cents a day (with a patient compliance of $60 \%$ ) the market value would increase from US\$1.1billion to $\$ 1.7$ billion by 2017 . With regard to self-pay, for a treatment that costs 50 cents a day (with a patient compliance of $60 \%$ ), 1.6 million patients (15\%) are able to afford treatment in 2007 (from 20\% of the household health-care budget). This is equivalent to a market value of $\$ 171$ million. Over the next decade, the proportion able to afford treatment is predicted to more than double to $33 \%$, increasing the market size to 5 million people, and market value to $\$ 550$ million. Of note is that the growth of the affordable market (compound annual growth rate of $12.4 \%$ per annum) far outweighs the growth of prevalence (2.3\% per annum), reflecting the importance of increasing affluence on patient access. With regard to the effect of reimbursement, if $30 \%$ reimbursement was granted, then an additional 1.9 million patients would be able to afford treatment in 2007 (one-third of all diagnosed diabetics), and the market size (patients able to afford treatment) and market value would double to 3.5 million patients and $\$ 373$ million, respectively. This analysis refers to diagnosed diabetics and does not include those who have diabetes but are unaware (undiagnosed). Source: Global Demographics Healthcare Ltd. 